Journal Club

Editor's Note: These short, critical reviews of recent papers in the Journal, written exclusively by graduate students or postdoctoral fellows, are intended to summarize the important findings of the paper and provide additional insight and commentary. For more information on the format and purpose of the Journal Club, please see http://www.jneurosci.org/misc/ifa_features.shtml.

\title{
Interactions between Resting-State and Task-Evoked Brain Activity Suggest a Different Approach to fMRI Analysis
}

\author{
Dana Mastrovito \\ Center for Molecular and Behavioral Neural Science, Rutgers University, Newark, New Jersey 07102 \\ Review of $\mathrm{He}$
}

The most widely used analytic approach in functional magnetic resonance imaging (fMRI) research is the general linear model (GLM). This approach is based on the idea that brain regions involved in performing a task can be discovered by subtracting the activity measured during task performance from that of a control condition. Typically, both experimental and control trials are performed many times and trials of each type are averaged together in an effort to increase the signalto-noise ratio. In some cases, the brain's activity measured in the absence of a task or experimental stimuli (so called "resting state") is used as a control. In other cases, the control may differ from the experimental condition only in the cognitive feature to be studied. In either case, the analysis relies on the assumption that brain activity measured in task and control conditions sums linearly. In order for this assumption to be valid, each experimental condition must evoke additional physiological activity that does not influence activity related to other conditions, a concept known as "pure insertion." Although most studies are based on this assumption, there is a growing body of

Received June 18, 2013; accepted July 3, 2013.

This material is based upon work supported by the National Science Foundation Graduate Research Fellowship under grant number DGE1313667. I thank Drs. Denis Pare' and Stephen Hanson.

Correspondence should be addressed to Dana Mastrovito, Center for Molecular and Behavioral Neural Science, Rutgers University, 197 University Avenue, Newark, NJ 07102. E-mail: dana.mastrovito@rutgers.edu.

DOI:10.1523/JNEUROSCI.2580-13.2013

Copyright $\odot 2013$ the authors $\quad 0270-6474 / 13 / 3312912-03 \$ 15.00 / 0$ evidence demonstrating that brain activity does not sum linearly. Indeed, there is evidence of nonlinear and complex behavior on all levels of neuronal function from the bursting patterns of single cells to complex interactions of cortical ensembles (Korn and Faure, 2003). More than 15 years ago, Friston et al. (1996) used a factorial experimental design to challenge the assumptions of pure insertion. This work demonstrated that subtractive experimental designs lead to erroneous conclusions due to nonlinear interactions between brain regions and experimental conditions. Interactions between different experimental conditions, which have been observed in many studies, suggest that measured brain activity should be viewed as the product of a dynamic system whose activity at any point is influenced by previous brain activity. Therefore, consideration of the interactions between brain activity across different functional states is critical to understanding brain dynamics.

Many researchers ceased using restingstate as a control condition after the discovery of functionally relevant structure in resting-state brain activity (Biswal et al., 1995). Since then, an increasing amount of research has gone into characterizing resting-state activity. It has been shown that patterns of correlated brain activity during rest are consistent across individuals, and predictive of disease states when abnormal. Additionally, a network of brain regions has been identified that exhibit increased activity at rest and de- creased activity during task performance, called the default mode network (DMN). Despite these findings, and even though task-related metabolic increases account for only $5 \%$ of total neural metabolism, there is currently no consensus on how to interpret metabolic activity measured in resting-state (Raichle and Mintun, 2006). Resting-state is often described as the spontaneous activity of the brain. Other definitions focus on activity associated with self-referential thoughts, or awareness of sensory input, and environmental changes.(Fransson, 2005; Fox and Raichle, 2007) The critical point is that if this activity is consistent and persists independently of task performance, it should subtract out of any GLM analysis leaving only the brain activity of interest. However, many studies (see Northoff et al., 2010 for review) have shown that brain activity measured in the resting state and task-evoked activity influence one another.

Evidence of the interaction between ongoing and evoked brain activity came from a comparison of variance in spontaneous and evoked activity published recently in The Journal of Neuroscience by Biyu He. This author points out that if ongoing and task-evoked activity sum linearly without interaction, then averaging across trials would suppress the variability in ongoing activity, revealing the "true" task-evoked response. However, if there is a positive or negative interaction between spontaneous and task-evoked activity, then single trial brain responses would be partially canceled out when averaged to- 
gether. The author used the law of variance of sums to challenge the assumption that spontaneous and task-evoked activity vary independently of one another. This law states that the sum of the variance of two or more random variables is equal to the sum of the variance of each variable only if the variables are independent. If they are not independent, the sum of their variances also depends on their covariances. With two variables (ongoing brain activity and task-evoked activity), there are three possibilities each resulting in a different relationship between the sum of their variances: no interaction between the two variables, positive interaction, and negative interaction. Both positive interaction and no interaction would result in greater variance in across-trial taskassociated activity than during rest. Negative interaction, on the other hand, would cause a decrease in variance associated with task activity compared with rest. Subjects were asked to perform a target detection task interspersed with resting periods. For whole brain analysis and a separate analysis on 33 regions of interest (ROIs), variances were calculated as the square of the SDs of trial-to-trial measurements during rest and target detection periods. The data showed a decrease in variability in all 33 ROIs (significant decrease at $p<0.05$ in 19 ROIs and at the population level at $p<1 \mathrm{e}-16)$ and in $86.8 \%$ of voxels in the whole brain analysis. This result is strong evidence of a negative interaction between spontaneous and task-evoked activity. Biyu He's study represents a novel mathematical characterization of the interaction between ongoing brain activity and that measured during task performance. Although these results were obtained using a target detection task, many others have demonstrated such interactions using a variety of other experimental protocols.

Several studies have yielded evidence that task-evoked activation of cortical networks shape the pattern of correlated activity at rest. In one study, it was shown that training required for a shape discrimination task induced a negative correlation in the resting-state between visual cortex and fronto-parietal attentional areas whose activity was independent before training (Lewis et al., 2009). These negative correlations corresponded with improved perceptual learning and did not extend to other networks. Such results suggest that the DMN functions as a filter for unattended sensory information, becoming less important as training progresses (Lewis et al., 2009). Albert et al.
(2009) obtained similar results using a motor learning paradigm. Therefore, resting-state activity may contain physiological evidence of network level neuroplasticity arising from experience-driven coactivation of cortical circuitries. While these studies provide evidence that taskevoked brain activity has an influence on the brain at rest, there are also indications that ongoing brain activity influences that evoked by experimental manipulation.

Several studies have demonstrated the influence of ongoing brain activity on task-performance. One such study, showed that pain intensity perception could be predicted by preceding levels of brain activity in the medial thalamus, lateral fronto-parietal areas, posterior cingulate, precuneus and tempero-pariatel cortices (Boly et al., 2007). Mennes et al. (2010) demonstrated that interindividual differences in resting-state functional connectivity could be used to predict differences in task-induced BOLD activity in a flanker task paradigm. In another study, Weissman et al. (2006) used a visual attention task to show that attentional lapses begin with reduced prestimulus activity in the anterior cingulate and less deactivation in the DMN, leading to system wide changes in stimulus-evoked activity. Therefore, regionally specific variability in resting activity also predicts changes in behavioral performance and task-evoked brain activity.

This growing body of evidence of interactions between brain activity across experimental conditions motivates using more sophisticated approaches to fMRI data analysis. Biyu He suggests that reduction of variability during task performance may facilitate sensory processing and may be an additional informationtransmitting feature in the brain. He recommends several alternative analysis approaches for fMRI such as multivariate pattern analysis, which takes into consideration both the across-trial mean response and trial-to-trial variability. Multivariate approaches also have the advantage of accounting for spatial correlations across voxels rather than erroneously assuming that each voxel's activity is independent. Additionally, as the author points out, his results are consistent with an alternative view of brain function as a "dynamic system selecting its trajectory based on context and tightening the trajectory when performing a specific task" as apposed to an independent amalgam of ongoing and evoked activity. Bjyu He's study, and others that demonstrate interactions between ongoing brain activity and experimentally induced activity, collectively make a case for fMRI analysis using state space modeling (SSM). Because of its ability to model spatial and temporal interactions, SSM provides an excellent framework for understanding the sequential changes in neural systems underlying behavior. SSM describes the brain as a dynamic system comprised of regional interactions, which can be described at any time by a point in multidimensional space. In a state-space model, temporal evolution of brain activity is determined by a smooth trajectory described by a transition matrix that is dependent on previous states, activity in other brain areas, and perturbations due to external stimuli. In this way, the transition matrix encapsulates both endogenous and exogenous influences. Although the computational requirements of this kind of analysis are significant and require thoughtful approaches to data dimensionality reduction, several groups have successfully used such models for fMRI analysis. Janoos et al. (2011) used SSM to distinguish between a control group and one with developmental dyscalculia (a mathematical disability) in a multiplication task. Unlike traditional analysis methods however, SSM also allowed them to characterize the spatio-temporal variations both between and within populations. Gössl et al. (2000) applied the technique to $\mathrm{fMRI}$ data collected during visual and auditory stimulation and showed that the influence and spatial extent of activation changed over the course of the experiment. Therefore, using more sophisticated multidimensional methods of analysis it is possible to move beyond an understanding of brain function that merely assigns participation of brain regions to cognitive abilities. These models allow for deeper investigation of temporal dynamics that underly shifts between varying degrees of consciousness, and attentional focus as well as the dependence of psychiatric disorders on the temporal dynamics of brain activity.

\section{References}

Albert NB, Robertson EM, Miall RC (2009) The resting human brain and motor learning. Curr Biol 19:1023-1027. CrossRef Medline

Biswal B, Yetkin FZ, Haughton VM, Hyde JS (1995) Functional connectivity in the motor cortex of resting human brain using echoplanar MRI. Magn Reson Med 34:537-541. CrossRef Medline

Boly M, Balteau E, Schnakers C, Degueldre C, Moonen G, Luxen A, Phillips C, Peigneux P, Maquet P, Laureys S (2007) Baseline brain activity fluctuations predict somatosensory perception in humans. Proc Natl Acad Sci U S A 104:12187-12192. CrossRef Medline 
Fox MD, Raichle ME (2007) Spontaneous fluctuations in brain activity observed with functional magnetic resonance imaging. Nat Rev Neurosci 8:700-711. CrossRef Medline

Fransson P (2005) Spontaneous low-frequency BOLD signal fluctuations: an fMRI investigation of the resting-state default mode of brain function hypothesis. Hum Brain Mapp 26: 15-29. CrossRef Medline

Friston KJ, Price CJ, Fletcher P, Moore C, Frackowiak RS, Dolan RJ (1996) The trouble with cognitive subtraction. Neuroimage 4:97-104. CrossRef Medline

Gössl C, Auer DP, Fahrmeir L (2000) Dynamic models in fMRI. Magn Reson Med 43:72-81. CrossRef Medline

$\mathrm{He}$ BJ (2013) Spontaneous and task-evoked brain activity negatively interact. J Neurosci 33:4672-4682. CrossRef Medline

Janoos F, Singh S, Machiraju R, Wells WM, Mórocz IA (2011) State-space models of mental processes from fMRI. Inf Process Med Imaging 22:588-599. Medline

Korn H, Faure P (2003) Is there chaos in the brain? II. Experimental evidence and related models. C R Biol 326:787-840. CrossRef Medline

Lewis CM, Baldassarre A, Committeri G, Romani GL, Corbetta M (2009) Learning sculpts the spontaneous activity of the resting human brain. Proc Natl Acad Sci U S A 106:17558-17563. CrossRef Medline

Mennes M, Kelly C, Zuo XN, Di Martino A, Biswal BB, Castellanos FX, Milham MP (2010) Interindividual differences in resting-state functional connectivity predict task-induced BOLD activity. Neuroimage 50:1690-1701. CrossRef Medline

Northoff G, Qin P, Nakao T (2010) Reststimulus interaction in the brain: a review. Trends Neurosci 33:277-284. CrossRef Medline

Raichle ME, Mintun MA (2006) Brain work and brain imaging. A comprehensive review of brain circulation and metabolism, its relationship to neurophysiology and functional brain imaging signals. Annu Rev Neurosci 29: 449-476. CrossRef Medline

Weissman DH, Roberts KC, Visscher KM, Woldorff MG (2006) The neural bases of momentary lapses in attention. Nat Neurosci 9:971-978. CrossRef Medline 